\title{
Fluoride Contamination of Groundwater and Health Hazard in Central India
}

\author{
Nohar Singh Dahariya, Keshaw Prakash Rajhans, Ankit Yadav, Shobhana Ramteke, \\ Bharat Lal Sahu, Khageshwar Singh Patel ${ }^{*}$ \\ School of Studies in Chemistry/Environmental Science, Pt Ravishankar Shukla University, Raipur, India \\ Email: *patelkhageshwarsingh@gmail.com
}

Received 28 October 2015; accepted 7 December 2015; published 10 December 2015

Copyright (C) 2015 by authors and Scientific Research Publishing Inc.

This work is licensed under the Creative Commons Attribution International License (CC BY).

http://creativecommons.org/licenses/by/4.0/

c) (i) Open Access

\begin{abstract}
The basic bed rocks of central India are contaminated with fluorite minerals. The overuse of groundwater for irrigation causes increased mineralization of $F^{-}$in the groundwater. This contaminated groundwater is widely used for drinking and other household purposes. The excess $F^{-}$is excreted through urine of animals. In this work, the exposure of contaminated groundwater in domestic animals of Dongargarh city, Chhattisgarh, India is studied. The symptoms of fluorosis diseases in the domestic animals i.e. cattle and buffalo are surveyed. The quality and sources of the contaminants of the groundwater are discussed.
\end{abstract}

\section{Keywords}

\section{Groundwater, Fluoride, Exposure, Fluorosis}

\section{Introduction}

Abnormal levels of $\mathrm{F}^{-}$in the groundwater are common in India due to weathering of the fractured hard rock pegmatite veins composing of minerals viz. topaz, fluorite fluorapatite, villuamite, cryolite, ferro magnesium silicate, etc. [1]. The $\mathrm{F}^{-}$contamination of groundwater in several states of the country was reported [2]-[21]. Millions of people and animals were exposed to excessive amount of $\mathrm{F}^{-}$through drinking water contaminated from geogenic and anthropogenic sources, suffering with various types of fluoride diseases [22]-[31]. The goal of this work is to study $\mathrm{F}^{-}$contamination of the groundwater and its exposure in domestic animals i.e. cattle and buffalo of Dongargarh city, India.

"Corresponding author.

How to cite this paper: Dahariya, N.S., Rajhans, K.P., Yadav, A., Ramteke, S., Sahu, B.L. and Patel, K.S. (2015) Fluoride Contamination of Groundwater and Health Hazard in Central India. Journal of Water Resource and Protection, 7, 1416-1428. http://dx.doi.org/10.4236/jwarp.2015.717115 


\section{Materials and Methods}

\subsection{Study Area}

Dongargarh $\left(21.18842^{\circ} \mathrm{N}\right.$ and $\left.80.75875^{\circ} \mathrm{E}\right)$ is a tourist city in central India with population of 0.1 million inclusive of neighboring villages. The town was settled near majestic mountains. The contaminated groundwater is widely used for drinking, cooking, washing and agricultural purposes. Four minerals i.e. oligoclase, rectorite, kaolinite and feldspar have been identified in the studied area. Feldspar is one of the most dominant mineral constituents of all the above-mentioned rocks, which is highly susceptible to chemical weathering and produces various types of clay minerals [32].

\subsection{Sample Collection}

Forty eight groundwater samples were collected from the tube wells of the Dongargarh city from $\approx 100 \mathrm{~km}^{-2}$ area in the post monsoon (January) and pre monsoon (May) period, 2014 by using established method, Figure 1

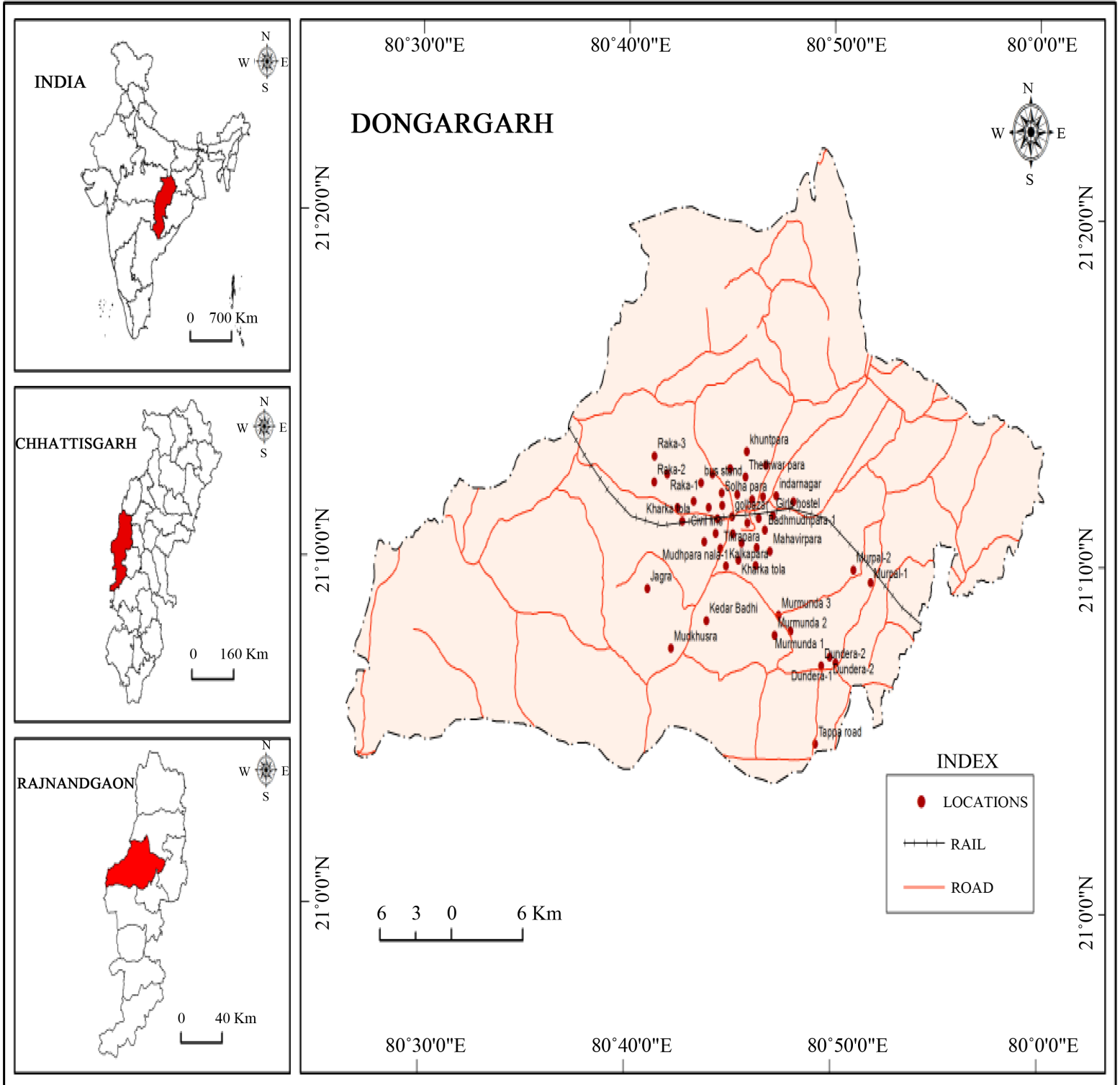

Figure 1. Representation of sampling locations in Dongargarh area, Chhattisgarh, India. 
[33]. The groundwater sample was stored in 1-L cleaned polyethylene bottle. The physical parameters i.e. pH, temperature (T), electrical conductivity (EC), dissolved oxygen (DO) and reduction potential (RP) of the water were analyzed at the spot. The water samples were dispatched to the laboratory and preserved in the deep freezer.

The first morning urine sample $(100 \mathrm{ml})$ was collected in plastic bottles containing $0.2 \mathrm{~g}$ EDTA. Total 40 urine samples of cattle and buffalo were collected in January, 2014. They were shipped to the laboratory in insulated container at about $4{ }^{\circ} \mathrm{C}$ and refrigerated at $-20^{\circ} \mathrm{C}$ until use.

\subsection{Analysis}

The total dissolved solid (TDS) value was determined by evaporation of the filtered water sample (through glass fiber filter) by drying at constant weight. The total hardness (TH) and total alkalinity (TA) values were analyzed by titration methods [34]. The $\mathrm{F}^{-}$content was analyzed by using Metrohm ion meter-781 in the presence of 1:1 total ion strength adjustment buffer (TISAB). The buffer was prepared by adding $58 \mathrm{~g} \mathrm{NaCl}+5 \mathrm{~g}$ CDTA (trans1 , 2, NNNN, cyclodiamine tetra acetic acid) $+57 \mathrm{ml}$ glacial acetic acid and deionized water by adjusting $\mathrm{pH}$ value to 5.5 with $8 \mathrm{~N} \mathrm{NaOH}$ in 1-L volumetric flask.

The ion (i.e. $\mathrm{Cl}^{-}, \mathrm{NO}_{3}^{-}, \mathrm{SO}_{4}^{2-}, \mathrm{NH}_{4}^{+}, \mathrm{Na}^{+}, \mathrm{K}^{+}, \mathrm{Mg}^{2+}$ and $\mathrm{Ca}^{2+}$ ) content of the water was analyzed by Dionex-1100 ion chromatography equipped with the anion and cation columns.

The water quality index (WQI) of the groundwater was computed by using the weighed arithmetic method. The value of 6 parameters i.e. $\mathrm{pH}, \mathrm{DO}, \mathrm{EC}$, TDS, TA and $\mathrm{NO}_{3}^{-}$was used in calculation of the WQI with the help of following expression.

$$
W Q I=\sum q_{n} W_{n} / \sum W_{n}
$$

where:

$$
q_{n}=100\left(V_{o}-V_{\text {io }}\right) /\left(V_{s}-V_{\text {io }}\right)
$$

$q_{n}=$ Quality rating of the nth water quality parameter;

$V_{n}=$ Estimated value of the nth parameter of a given water;

$S_{n}=$ Standard permissible value of the nth parameter;

$V_{\text {io }}=$ Ideal value of the nth parameter of pure water (i.e., 0 for all other parameters except $\mathrm{pH}$ and dissolved oxygen (7.0 and $14.6 \mathrm{mg} / \mathrm{L}$, respectively);

$W_{n}=$ Unit weight for the nth parameter;

$K=$ Proportionality constant.

Multivariate statistical analysis such as factor analysis (FA) was employed for the source apportionment. The windows statistical software Statistica-7.1 was used for the multivariate statistical calculation.

\section{Results and Discussion}

\subsection{Geological Characteristics of Tube Well}

The geological characteristics of the tube wells is summarized in Table 1 . The tube wells of the studied area lie in the deeper zone, ranging from 45 - $110 \mathrm{~m}$. The life was ranged from 2 - $50 \mathrm{Yr}$ old. Tube wells are recharged by rain and runoff water during rainy season. The water table is varied from $20-50 \mathrm{~m}$, depending on seasons and water uses. The higher $\mathrm{T}$ value for deeper tube well was observed due to geothermal energy. In turn, the higher DO value of shallow tube wells was marked.

\subsection{Physical Characteristics of Groundwater}

The physical characteristics of the groundwater in the post monsoon period is shown in Table 1 . The value of $\mathrm{pH}, \mathrm{DO}, \mathrm{T}, \mathrm{RP}, \mathrm{EC}, \mathrm{TDS}, \mathrm{TA}$ and TH of groundwater located in 48 tube wells was ranged from 6.0 - 8.1, 8.4 $9.2 \mathrm{mg} / \mathrm{L}, 20.0^{\circ} \mathrm{C}-25.0^{\circ} \mathrm{C}, 237-330 \mathrm{mV}, 221-1938 \mu \mathrm{S} / \mathrm{cm}, 342-2598 \mathrm{mg} / \mathrm{L}, 128-659 \mathrm{mg} / \mathrm{L}$ and $99-687$ $\mathrm{mg} / \mathrm{L}$ with mean value of $7.2 \pm 0.1,8.9 \pm 0.1,23.0 \pm 0.3,285 \pm 5 \mathrm{mV}, 861 \pm 96 \mu \mathrm{S} / \mathrm{cm}, 1138 \pm 121 \mathrm{mg} / \mathrm{L}, 383 \pm$ $47 \mathrm{mg} / \mathrm{L}$ and $344 \pm 37 \mathrm{mg} / \mathrm{L}$, respectively. Two ions i.e. $\mathrm{Na}^{+}$and $\mathrm{SO}_{4}^{2-}$ were found to be responsible for contributing the EC value of the water. The DO, EC, TDS, TA and TH value of water was found to be higher than recommended value of $4.0 \mathrm{mg} / \mathrm{L}, 300 \mu \mathrm{S} / \mathrm{cm}, 500 \mathrm{mg} / \mathrm{L}, 120 \mathrm{mg} / \mathrm{L}$ and $300 \mathrm{mg} / \mathrm{L}$, respectively [35] [36]. The 
Table 1. Characteristics of tube well and groundwater in the post monsoon period, 2014.

\begin{tabular}{|c|c|c|c|c|c|c|c|c|}
\hline S. No. & Location & Age, $\mathrm{Yr}$ & Depth, m & $\mathrm{T}^{\circ} \mathrm{C}$ & $\mathrm{pH}$ & $\mathrm{EC}, \mu \mathrm{S} / \mathrm{cm}$ & $\mathrm{RP}, \mathrm{mV}$ & $\mathrm{DO}, \mathrm{mg} / \mathrm{L}$ \\
\hline 1 & Mahavir Para & 20 & 75 & 21 & 7.3 & 831 & 283 & 8.8 \\
\hline 2 & Goal Bajar & 1 & 82 & 21 & 6.1 & 1017 & 310 & 8.9 \\
\hline 3 & Bhagat Shih Ward & 3 & 75 & 21 & 8.1 & 967 & 237 & 8.6 \\
\hline 4 & Civil Line & 7 & 69 & 22 & 6.2 & 991 & 311 & 8.8 \\
\hline 5 & Shubhash Ward & 1 & 90 & 22 & 6.5 & 1013 & 319 & 8.9 \\
\hline 6 & Thana Chowk & 7 & 69 & 22 & 6.4 & 1139 & 313 & 8.9 \\
\hline 7 & Kachari Chowk & 12 & 75 & 21 & 6.6 & 603 & 304 & 8.8 \\
\hline 8 & Thethwar Para & 12 & 82 & 21 & 6.5 & 344 & 309 & 8.7 \\
\hline 9 & BUS Stand & 25 & 75 & 22 & 6.0 & 609 & 319 & 8.8 \\
\hline 10 & Solh Para & 2 & 105 & 22 & 6.1 & 982 & 330 & 8.8 \\
\hline 11 & Ek-batti Char Rasta & 10 & 66 & 23 & 8.0 & 733 & 265 & 9.1 \\
\hline 12 & Kumhar Para & 15 & 54 & 22 & 6.5 & 385 & 269 & 9.0 \\
\hline 13 & School & 10 & 69 & 22 & 6.3 & 276 & 297 & 9.2 \\
\hline 14 & Kumhar Para & 1 & 75 & 22 & 6.9 & 349 & 298 & 8.8 \\
\hline 15 & Ambedkar Ward & 1 & 90 & 23 & 7.3 & 613 & 254 & 8.9 \\
\hline 16 & Station & 3 & 92 & 23 & 7.6 & 888 & 246 & 8.8 \\
\hline 17 & Bheem Nagar & 4 & 105 & 22 & 7.3 & 1451 & 254 & 8.7 \\
\hline 18 & Utkarsh Nagar & 8 & 110 & 22 & 7.3 & 1329 & 290 & 8.9 \\
\hline 19 & Kalka Para & 10 & 75 & 22 & 7.2 & 1331 & 293 & 8.7 \\
\hline 20 & Indra Nagar & 15 & 60 & 23 & 7.4 & 969 & 277 & 9.0 \\
\hline 21 & Rajiv Nagar & 6 & 62 & 21 & 7.1 & 1178 & 304 & 8.7 \\
\hline 22 & Sanjay Nagar & 10 & 66 & 22 & 7.2 & 1062 & 285 & 9.1 \\
\hline 23 & Khuta Para & 12 & 72 & 22 & 7.2 & 951 & 302 & 8.4 \\
\hline 24 & Danteshwari Para & 10 & 75 & 23 & 7.4 & 670 & 290 & 8.8 \\
\hline 25 & Kharka Tola & 10 & 69 & 22 & 7.9 & 1033 & 282 & 9.0 \\
\hline 26 & Tikra Para & 4 & 84 & 22 & 7.7 & 858 & 276 & 8.9 \\
\hline 27 & Kedar Badhi & 23 & 75 & 21 & 7.6 & 1014 & 286 & 8.8 \\
\hline 28 & Badhiya Tola & 30 & 82 & 23 & 7.1 & 1199 & 288 & 9.0 \\
\hline 29 & Raka Panchayat & 24 & 90 & 22 & 7.2 & 637 & 275 & 9.0 \\
\hline 30 & Raka-2 & 50 & 75 & 21 & 7.2 & 322 & 291 & 9.1 \\
\hline 31 & Raka-3 & 3 & 92 & 21 & 7.1 & 1202 & 291 & 8.9 \\
\hline 32 & Murmunda-1 & 4 & 84 & 22 & 7.2 & 817 & 292 & 8.8 \\
\hline 33 & Murmunda-2 & 5 & 75 & 22 & 7.2 & 687 & 285 & 9.0 \\
\hline 34 & Murmunda-3 & 3 & 69 & 22 & 7.3 & 735 & 283 & 9.1 \\
\hline 35 & Murpal-1 & 10 & 45 & 22 & 7.3 & 539 & 276 & 8.9 \\
\hline 36 & Murpal-2 & 4 & 56 & 23 & 7.2 & 625 & 299 & 9.0 \\
\hline 37 & Murpal-3 & 6 & 64 & 23 & 7.4 & 1938 & 277 & 8.7 \\
\hline 38 & Jagra & 2 & 75 & 22 & 7.4 & 221 & 272 & 8.9 \\
\hline 39 & Badhmudh Par-1 & 5 & 72 & 22 & 7.6 & 623 & 278 & 8.6 \\
\hline 40 & Badhmudhpar-2 & 21 & 72 & 21 & 7.5 & 707 & 276 & 8.7 \\
\hline 41 & Mudhpara Nala-1 & 7 & 75 & 22 & 7.8 & 1053 & 287 & 9.0 \\
\hline 42 & Mudhpara Nala-2 & 12 & 75 & 21 & 7.8 & 1057 & 272 & 8.9 \\
\hline 43 & Dundera-1 & 5 & 66 & 23 & 7.7 & 895 & 275 & 9.0 \\
\hline 44 & Dundera-2 & 20 & 69 & 22 & 7.6 & 1057 & 280 & 8.9 \\
\hline 45 & SBI Bank & 10 & 90 & 22 & 7.2 & 605 & 294 & 8.9 \\
\hline 46 & Tappa Road & 20 & 75 & 22 & 7.8 & 785 & 262 & 9.0 \\
\hline 47 & Mudhkhusra & 10 & 66 & 21 & 7.3 & 681 & 269 & 9.1 \\
\hline 48 & Shivnikunj & 10 & 75 & 22 & 7.7 & 1373 & 272 & 9.0 \\
\hline
\end{tabular}


RP value of water was marked to be just of a half of recommended value of $600 \mathrm{mV}$.

\subsection{Chemical Characteristics of Groundwater}

The chemical characteristics of the groundwater in the post monsoon period is shown in Table 2. The concentration of ions i.e. $\mathrm{F}^{-}, \mathrm{Cl}^{-}, \mathrm{NO}_{3}^{-}, \mathrm{SO}_{4}^{2-}, \mathrm{NH}_{4}^{+}, \mathrm{Na}^{+}, \mathrm{K}^{+}, \mathrm{Mg}^{2+}$ and $\mathrm{Ca}^{2+}$ in the groundwater of 48 tube wells was ranged from $2.0-10.3,43-408,48-152,12-161,10-144,8.0-75,3.0-25,9.0-57$ and $24-172 \mathrm{mg} / \mathrm{L}$ with mean value of $4.9 \pm 0.5,121 \pm 22,75 \pm 8,47 \pm 9,55 \pm 11,41 \pm 5,6.1 \pm 1.5,28 \pm 3$ and $87 \pm 9 \mathrm{mg} / \mathrm{L}$, respectively. The $\mathrm{F}^{-}$and $\mathrm{NO}_{3}^{-}$crossed the recommended limit of 1.5 and $45 \mathrm{mg} / \mathrm{L}$, respectively in the water of all tube wells [35] [36]. However, $\mathrm{Mg}$ and Ca concentration was above recommended limit of 30 and $75 \mathrm{mg} / \mathrm{L}$ in the $33 \%$ and $67 \%$ tube wells [35] [36].

\subsection{Seasonal Variation and Sources of Fluoride}

The chemical data for the pre monsoon period, 2014 is presented in Table 3. The variation of physical and chemical parameters of the water in the pre monsoon period (May 2014) is presented in Figure 2. The value of $\mathrm{pH}, \mathrm{EC}, \mathrm{TDS}, \mathrm{TA}, \mathrm{TH}, \mathrm{F}, \mathrm{Na}, \mathrm{Mg}$ and $\mathrm{Ca}$ was found to be increased $\geq 30 \%$, may be due to increase of water temperature, $\approx 4^{\circ} \mathrm{C}$, and deduction of water level up to $50 \mathrm{~m}$. The $\mathrm{F}^{-}$with the metals i.e. $\mathrm{Na}^{+}, \mathrm{Mg}^{2+}$ and $\mathrm{Ca}^{2+}$ had good correlation $(\mathrm{r}=0.78-0.85)$, indicating origin from the rock weathering, Figure 3. Other ions (i.e. $\mathrm{Na}^{+}$, $\mathrm{Mg}^{2+}, \mathrm{Ca}^{2+}, \mathrm{Cl}^{-}$and $\mathrm{SO}_{4}^{2-}$ ) among themselves had fare correlation, suggesting origin from multiple sources, Table 4. However, two ions i.e. $\mathrm{NH}_{4}^{+}$and $\mathrm{NO}_{3}^{-}$had good correlation, originating from similar anthropogenic sources.

\subsection{Factor Analysis}

The factor analysis of data has extracted six factors which explained $77.25 \%$ of the variance in the data set. The loadings of variables, eigenvalues and cumulative variance for each factor are shown in Table 5. Factor- 1 accounted for $32.66 \%$ of the total variance with high positive loadings of $\mathrm{Ca}^{2+}, \mathrm{Mg}^{2+}, \mathrm{F}^{-}$and $\mathrm{TH}$. This factor suggests the role of dissolution/precipitation processes of some minerals such as $\mathrm{CaF}_{2}$ and $\mathrm{CaCO}_{3}$. Factor-2 accounted for $13.31 \%$ of representation with strong positive loading of $\mathrm{pH}$ which is negatively correlated with redox potential (RP). This factor suggests occurrence of redox processes which determine the acidic or alkaline nature of groundwater. Factor-3 represents $10.80 \%$ of the total variance with strong positive loadings of $\mathrm{NH}_{4}^{+}$ and $\mathrm{NO}_{3}^{-}$. This factor loadings shows the anthropogenic influences on these parameters. Factor- 4 yielded $8.39 \%$ of the total variance with strong positive loadings of $\mathrm{SO}_{4}^{2-}$ and $\mathrm{Na}^{+}$suggesting mineral weathering. Factor-5 accounted for $6.32 \%$ of the total variance with high positive loading of $\mathrm{K}^{+}$. In groundwater, $\mathrm{K}$ could proceed from fertilizers or weathering of K-feldspar. Factor- 6 accounted for $5.66 \%$ of the total variance with a high positive value of temperature $(\mathrm{T})$. The $\mathrm{T}$ value is a variable which controls many reactions.

\subsection{Water Quality Index}

The WQI of the water in the post monsoon period was ranged from $22-226$ with mean value of $97 \pm 12$. The value of TDS, TA, TH, $\mathrm{F}^{-}$and $\mathrm{NO}_{3}^{-}$in the water of all tube wells was found above permissible limits of 500, 120, 300, 1.5 and $45 \mathrm{mg} / \mathrm{L}$, respectively [35] [36]. However, in the pre monsoon period, the value of $\mathrm{F}^{-}, \mathrm{Mg}^{2+}$ and $\mathrm{Ca}^{2+}$ crossed significantly the prescribed permissible limit of 1.5, 30 and 75, making water unsafe for drinking purposes.

\subsection{Fluoride Toxicities}

Chronic ingestion of fluoride water in endemic areas leads to development of fluorosis in the animal e.g. dental discoloration, difficulty in mastication, bony lesions, lameness, disability and mortality [37]. In lower age group, the lesion of teeth, skin, hair and nails were frequently observed. Fluoride enters the animal body mainly through the intake of water and quickly absorbed in the gastrointestinal tract. The excess $\mathrm{F}^{-}$is excreted largely through the urine. The survey for the fluorosis in domestic animals (3 - 15 Yr) in the Dongargarh area, Rajnandgaon, Chhattisgarh, India was carried out in January 2014. A total of 40 domestic animals were screened for prevalence of various types of fluorosis i.e. lesion, dental, horn skin and toe fluorosis. The $\mathrm{F}^{-}$concentration 
Table 2. Chemical characteristics of groundwater in post monsoon period, 2014, mg/L.

\begin{tabular}{|c|c|c|c|c|c|c|c|c|c|c|c|c|}
\hline S. No. & TDS & $\mathrm{TA}$ & $\mathrm{TH}$ & $F^{-}$ & $\mathrm{Cl}^{-}$ & $\mathrm{NO}_{3}^{-}$ & $\mathrm{SO}_{4}^{2-}$ & $\mathrm{NH}_{4}^{+}$ & $\mathrm{Na}^{+}$ & $\mathrm{K}^{+}$ & $\mathrm{Mg}^{2+}$ & $\mathrm{Ca}^{2+}$ \\
\hline 1 & 1187 & 293 & 414 & 5.5 & 89 & 95 & 50 & 90 & 32 & 3.1 & 30 & 108 \\
\hline 2 & 1474 & 173 & 327 & 5.2 & 195 & 71 & 81 & 64 & 26 & 3.2 & 31 & 78 \\
\hline 3 & 1411 & 275 & 411 & 6.2 & 160 & 66 & 74 & 57 & 34 & 6.4 & 29 & 108 \\
\hline 4 & 1347 & 128 & 432 & 6.1 & 188 & 51 & 41 & 15 & 37 & 14 & 32 & 112 \\
\hline 5 & 1308 & 189 & 291 & 4.1 & 142 & 107 & 55 & 99 & 28 & 3.3 & 25 & 72 \\
\hline 6 & 1510 & 165 & 363 & 3.4 & 231 & 71 & 37 & 65 & 22 & 12 & 29 & 92 \\
\hline 7 & 918 & 171 & 297 & 3.9 & 85 & 67 & 28 & 63 & 26 & 3.2 & 25 & 74 \\
\hline 8 & 694 & 128 & 111 & 2.5 & 60 & 112 & 23 & 94 & 20 & 15 & 11 & 26 \\
\hline 9 & 933 & 262 & 225 & 2.1 & 138 & 53 & 62 & 18 & 25 & 3.1 & 19 & 56 \\
\hline 10 & 1719 & 177 & 426 & 6.1 & 195 & 145 & 63 & 133 & 33 & 3.2 & 36 & 106 \\
\hline 11 & 886 & 275 & 123 & 2.2 & 92 & 53 & 68 & 39 & 39 & 12 & 11 & 30 \\
\hline 12 & 537 & 153 & 171 & 2.9 & 60 & 59 & 18 & 34 & 26 & 3.4 & 15 & 42 \\
\hline 13 & 550 & 153 & 99 & 3.4 & 56 & 72 & 17 & 69 & 34 & 3.5 & 9 & 24 \\
\hline 14 & 545 & 153 & 120 & 4.7 & 59 & 73 & 16 & 66 & 15 & 3.6 & 10 & 30 \\
\hline 15 & 822 & 293 & 246 & 3.9 & 89 & 65 & 29 & 52 & 17 & 3.7 & 18 & 64 \\
\hline 16 & 982 & 549 & 297 & 4.3 & 85 & 70 & 34 & 61 & 43 & 3.8 & 23 & 76 \\
\hline 17 & 1876 & 647 & 513 & 7.4 & 213 & 58 & 161 & 11 & 61 & 3.2 & 41 & 130 \\
\hline 18 & 1366 & 634 & 342 & 4.3 & 185 & 59 & 65 & 12 & 34 & 3.3 & 26 & 88 \\
\hline 19 & 1892 & 610 & 447 & 7.7 & 220 & 105 & 95 & 91 & 77 & 3.1 & 37 & 112 \\
\hline 20 & 1204 & 659 & 357 & 7.4 & 67 & 89 & 63 & 77 & 73 & 5.1 & 33 & 86 \\
\hline 21 & 1216 & 561 & 516 & 7.3 & 53 & 52 & 110 & 10 & 72 & 3.2 & 46 & 126 \\
\hline 22 & 1348 & 580 & 360 & 4.4 & 174 & 55 & 44 & 49 & 37 & 3.2 & 28 & 92 \\
\hline 23 & 1710 & 512 & 330 & 3.2 & 337 & 54 & 56 & 11 & 22 & 3.4 & 26 & 84 \\
\hline 24 & 825 & 433 & 348 & 4.9 & 57 & 51 & 22 & 48 & 36 & 3.5 & 28 & 88 \\
\hline 25 & 729 & 348 & 276 & 2.1 & 82 & 53 & 25 & 17 & 23 & 6.3 & 18 & 74 \\
\hline 26 & 968 & 531 & 291 & 3.8 & 124 & 53 & 35 & 11 & 45 & 21 & 21 & 76 \\
\hline 27 & 1287 & 549 & 330 & 3.9 & 149 & 105 & 26 & 94 & 45 & 3.2 & 24 & 86 \\
\hline 28 & 991 & 378 & 438 & 6.2 & 56 & 68 & 47 & 57 & 37 & 3.3 & 36 & 110 \\
\hline 29 & 957 & 305 & 282 & 4.1 & 75 & 89 & 41 & 85 & 17 & 9.1 & 24 & 70 \\
\hline 30 & 342 & 189 & 126 & 2.0 & 58 & 53 & 35 & 12 & 16 & 3.4 & 10 & 32 \\
\hline 31 & 1646 & 512 & 423 & 5.6 & 160 & 111 & 33 & 108 & 26 & 25 & 35 & 106 \\
\hline 32 & 1152 & 140 & 300 & 3.1 & 114 & 48 & 74 & 42 & 22 & 17 & 26 & 74 \\
\hline 33 & 1140 & 464 & 450 & 6.1 & 71 & 98 & 41 & 88 & 63 & 3.2 & 36 & 114 \\
\hline 34 & 909 & 397 & 294 & 3.1 & 85 & 82 & 12 & 77 & 27 & 3.3 & 26 & 72 \\
\hline 35 & 596 & 427 & 213 & 2.7 & 64 & 52 & 22 & 13 & 25 & 3.2 & 17 & 54 \\
\hline 36 & 729 & 366 & 279 & 6.1 & 75 & 57 & 41 & 12 & 65 & 3.5 & 23 & 70 \\
\hline 37 & 1188 & 390 & 435 & 6.1 & 75 & 49 & 104 & 11 & 63 & 6.2 & 39 & 106 \\
\hline 38 & 2598 & 622 & 654 & 9.1 & 408 & 88 & 89 & 80 & 58 & 12 & 54 & 164 \\
\hline 39 & 1042 & 512 & 534 & 7.4 & 43 & 67 & 12 & 56 & 69 & 6.3 & 38 & 140 \\
\hline 40 & 1014 & 512 & 321 & 3.1 & 54 & 145 & 26 & 132 & 52 & 12 & 27 & 80 \\
\hline 41 & 1200 & 427 & 315 & 4.1 & 107 & 99 & 26 & 86 & 31 & 3.7 & 27 & 78 \\
\hline 42 & 722 & 458 & 285 & 5.6 & 71 & 53 & 22 & 10 & 46 & 3.1 & 25 & 70 \\
\hline 43 & 1137 & 616 & 252 & 6.1 & 53 & 152 & 16 & 144 & 65 & 3.2 & 20 & 64 \\
\hline 44 & 1525 & 470 & 687 & 10.3 & 138 & 65 & 48 & 47 & 85 & 3.4 & 57 & 172 \\
\hline 45 & 657 & 299 & 348 & 5.1 & 57 & 53 & 15 & 17 & 58 & 3.4 & 26 & 90 \\
\hline 46 & 1199 & 360 & 342 & 4.5 & 124 & 81 & 52 & 72 & 42 & 3.5 & 28 & 86 \\
\hline 47 & 981 & 439 & 555 & 8.1 & 78 & 51 & 17 & 15 & 73 & 3.2 & 45 & 140 \\
\hline 48 & 1638 & 500 & 492 & 5.9 & 234 & 53 & 74 & 13 & 53 & 6.2 & 42 & 122 \\
\hline
\end{tabular}


Table 3. Characteristics of groundwater in pre monsoon period, May 2014.

\begin{tabular}{|c|c|c|c|c|c|c|c|c|c|c|c|}
\hline \multirow{2}{*}{ S. No. } & \multirow{2}{*}{$\mathrm{pH}$} & \multirow{2}{*}{ EC } & $\mathrm{F}^{-}$ & $\mathrm{Cl}^{-}$ & $\mathrm{NO}_{3}^{-}$ & $\mathrm{SO}_{4}^{2-}$ & $\mathrm{NH}_{4}^{+}$ & $\mathrm{Na}^{+}$ & $\mathrm{K}^{+}$ & $\mathrm{Mg}^{2+}$ & $\mathrm{Ca}^{2+}$ \\
\hline & & & \multicolumn{9}{|c|}{$\mathrm{mg} / \mathrm{L}$} \\
\hline 1 & 7.7 & 1064 & 6.8 & 71 & 58 & 58 & 58 & 41 & 2.9 & 36 & 126 \\
\hline 2 & 6.4 & 1302 & 6.4 & 156 & 43 & 94 & 41 & 33 & 2.9 & 37 & 91 \\
\hline 3 & 8.5 & 1238 & 7.6 & 128 & 40 & 86 & 36 & 43 & 5.9 & 35 & 126 \\
\hline 4 & 6.5 & 1268 & 7.5 & 150 & 31 & 48 & 10 & 47 & 12.9 & 38 & 131 \\
\hline 5 & 6.8 & 1297 & 5.0 & 114 & 65 & 64 & 63 & 36 & 3.0 & 30 & 84 \\
\hline 6 & 6.7 & 1458 & 4.2 & 185 & 43 & 43 & 42 & 28 & 11.0 & 35 & 108 \\
\hline 7 & 6.9 & 772 & 4.8 & 68 & 41 & 32 & 40 & 33 & 2.9 & 30 & 87 \\
\hline 8 & 6.8 & 440 & 3.1 & 48 & 68 & 27 & 60 & 25 & 13.8 & 13 & 30 \\
\hline 9 & 6.3 & 780 & 2.6 & 110 & 32 & 72 & 12 & 32 & 2.9 & 23 & 66 \\
\hline 10 & 6.4 & 1257 & 7.5 & 156 & 88 & 73 & 85 & 42 & 2.9 & 43 & 124 \\
\hline 11 & 8.4 & 938 & 2.7 & 74 & 32 & 79 & 25 & 50 & 11.0 & 13 & 35 \\
\hline 12 & 6.8 & 493 & 3.6 & 48 & 36 & 21 & 22 & 33 & 3.1 & 18 & 49 \\
\hline 13 & 6.6 & 353 & 4.2 & 45 & 44 & 20 & 44 & 43 & 3.2 & 11 & 28 \\
\hline 14 & 7.2 & 447 & 5.8 & 47 & 45 & 19 & 42 & 19 & 3.3 & 12 & 35 \\
\hline 15 & 7.7 & 785 & 4.8 & 71 & 40 & 34 & 33 & 22 & 3.4 & 22 & 75 \\
\hline 16 & 8.0 & 1137 & 5.3 & 68 & 43 & 39 & 39 & 55 & 3.5 & 28 & 89 \\
\hline 17 & 7.7 & 1857 & 9.1 & 170 & 35 & 187 & 7 & 77 & 2.9 & 49 & 152 \\
\hline 18 & 7.7 & 1701 & 5.3 & 148 & 36 & 75 & 8 & 43 & 3.0 & 31 & 103 \\
\hline 19 & 7.6 & 1704 & 9.5 & 176 & 64 & 110 & 58 & 98 & 2.9 & 44 & 131 \\
\hline 20 & 7.8 & 1240 & 9.1 & 54 & 54 & 73 & 49 & 93 & 4.7 & 40 & 101 \\
\hline 21 & 7.5 & 1508 & 9.0 & 42 & 32 & 128 & 6 & 91 & 2.9 & 55 & 147 \\
\hline 22 & 7.6 & 1359 & 5.4 & 139 & 34 & 51 & 31 & 47 & 2.9 & 34 & 108 \\
\hline 23 & 7.6 & 1217 & 3.9 & 270 & 33 & 65 & 7 & 28 & 3.1 & 31 & 98 \\
\hline 24 & 7.8 & 858 & 6.0 & 46 & 31 & 26 & 31 & 46 & 3.2 & 34 & 103 \\
\hline 25 & 8.3 & 1322 & 2.6 & 66 & 32 & 29 & 11 & 29 & 5.8 & 22 & 87 \\
\hline 26 & 8.1 & 1098 & 4.7 & 99 & 32 & 41 & 7 & 57 & 19.3 & 25 & 89 \\
\hline 27 & 8.0 & 1298 & 4.8 & 119 & 64 & 30 & 60 & 57 & 2.9 & 29 & 101 \\
\hline 28 & 7.5 & 1535 & 7.6 & 45 & 41 & 55 & 36 & 47 & 3.0 & 43 & 129 \\
\hline 29 & 7.6 & 815 & 5.0 & 60 & 54 & 48 & 54 & 22 & 8.4 & 29 & 82 \\
\hline 30 & 7.6 & 412 & 2.5 & 46 & 32 & 41 & 8 & 20 & 3.1 & 12 & 37 \\
\hline 31 & 7.5 & 1539 & 6.9 & 128 & 68 & 38 & 69 & 33 & 23.0 & 42 & 124 \\
\hline 32 & 7.6 & 1046 & 3.8 & 91 & 29 & 86 & 27 & 28 & 15.6 & 31 & 87 \\
\hline 33 & 7.6 & 879 & 7.5 & 57 & 60 & 48 & 56 & 80 & 2.9 & 43 & 133 \\
\hline 34 & 7.7 & 941 & 3.8 & 68 & 50 & 14 & 49 & 34 & 3.0 & 31 & 84 \\
\hline 35 & 7.7 & 690 & 3.3 & 51 & 32 & 26 & 8 & 32 & 2.9 & 20 & 63 \\
\hline 36 & 7.6 & 800 & 7.5 & 60 & 35 & 48 & 8 & 83 & 3.2 & 28 & 82 \\
\hline 37 & 7.8 & 2481 & 7.5 & 60 & 30 & 121 & 7 & 80 & 5.7 & 47 & 124 \\
\hline 38 & 7.8 & 283 & 11.2 & 326 & 54 & 103 & 51 & 74 & 11.0 & 65 & 192 \\
\hline 39 & 8.0 & 797 & 9.1 & 34 & 41 & 14 & 36 & 88 & 5.8 & 46 & 164 \\
\hline 40 & 7.9 & 905 & 3.8 & 43 & 88 & 30 & 84 & 66 & 11.0 & 32 & 94 \\
\hline 41 & 8.2 & 1348 & 5.0 & 86 & 60 & 30 & 55 & 39 & 3.4 & 32 & 91 \\
\hline 42 & 8.2 & 1353 & 6.9 & 57 & 32 & 26 & 6 & 58 & 2.9 & 30 & 82 \\
\hline 43 & 8.1 & 1146 & 7.5 & 42 & 93 & 19 & 92 & 83 & 2.9 & 24 & 75 \\
\hline 44 & 8.0 & 1353 & 12.7 & 110 & 40 & 56 & 30 & 108 & 3.1 & 68 & 201 \\
\hline 45 & 7.6 & 774 & 6.3 & 46 & 32 & 17 & 11 & 74 & 3.1 & 31 & 105 \\
\hline 46 & 8.2 & 1005 & 5.5 & 99 & 49 & 60 & 46 & 53 & 3.2 & 34 & 101 \\
\hline 47 & 7.7 & 872 & 10.0 & 62 & 31 & 20 & 10 & 93 & 2.9 & 54 & 164 \\
\hline 48 & 8.1 & 1757 & 7.3 & 187 & 32 & 86 & 8 & 67 & 5.7 & 50 & 143 \\
\hline
\end{tabular}


Table 4. Correlation matrix of ions.

\begin{tabular}{|c|c|c|c|c|c|c|c|c|c|}
\hline & F & $\mathrm{Cl}^{-}$ & $\mathrm{NO}_{3}^{-}$ & $\mathrm{SO}_{4}^{2-}$ & $\mathrm{NH}_{4}^{+}$ & $\mathrm{Na}^{+}$ & $\mathrm{K}^{+}$ & $\mathrm{Mg}^{2+}$ & $\mathrm{Ca}^{2+}$ \\
\hline $\mathrm{F}^{-}$ & 1 & & & & & & & & \\
\hline $\mathrm{Cl}^{-}$ & 0.24 & 1 & & & & & & & \\
\hline $\mathrm{NO}_{3}^{-}$ & 0.08 & 0.01 & 1 & & & & & & \\
\hline $\mathrm{SO}_{4}^{2-}$ & 0.38 & 0.49 & -0.14 & 1 & & & & & \\
\hline $\mathrm{NH}_{4}^{+}$ & 0.05 & -0.02 & 0.94 & -0.20 & 1 & & & & \\
\hline $\mathrm{Na}^{+}$ & 0.78 & -0.01 & 0.06 & 0.29 & -0.04 & 1 & & & \\
\hline $\mathrm{K}^{+}$ & -0.13 & 0.16 & 0.09 & -0.01 & 0.11 & -0.17 & 1 & & \\
\hline $\mathrm{Mg}^{2+}$ & 0.85 & 0.42 & 0.02 & 0.48 & 0.00 & 0.66 & -0.02 & 1 & \\
\hline $\mathrm{Ca}^{2+}$ & 0.83 & 0.43 & -0.01 & 0.41 & -0.03 & 0.64 & 0.00 & 0.97 & 1 \\
\hline
\end{tabular}

Table 5. Eigenvalues and factor loadings of Dongargarh groundwater samples.

\begin{tabular}{|c|c|c|c|c|c|c|}
\hline Variable & Factor-1 & Factor-2 & Factor-3 & Factor-4 & Factor-5 & Factor-6 \\
\hline Age & -0.18 & 0.07 & -0.06 & -0.15 & -0.45 & -0.66 \\
\hline Depth & -0.05 & -0.13 & 0.19 & 0.45 & 0.30 & -0.05 \\
\hline $\mathrm{T}$ & -0.09 & 0.17 & -0.06 & 0.00 & -0.25 & 0.82 \\
\hline $\mathrm{pH}$ & 0.07 & 0.94 & -0.04 & -0.01 & 0.01 & 0.00 \\
\hline EC & 0.24 & 0.17 & -0.10 & 0.69 & -0.16 & 0.11 \\
\hline $\mathrm{RP}$ & -0.07 & -0.85 & 0.12 & 0.11 & -0.09 & -0.08 \\
\hline DO & -0.11 & 0.06 & 0.05 & -0.51 & -0.24 & 0.26 \\
\hline TDS & 0.61 & -0.04 & 0.21 & 0.65 & 0.23 & 0.09 \\
\hline $\mathrm{TA}$ & 0.36 & 0.66 & 0.10 & 0.37 & -0.18 & 0.09 \\
\hline $\mathrm{TH}$ & 0.95 & 0.12 & -0.02 & 0.25 & 0.03 & -0.05 \\
\hline $\mathrm{F}^{-}$ & 0.90 & 0.11 & 0.06 & 0.08 & -0.11 & 0.12 \\
\hline $\mathrm{Cl}^{-}$ & 0.36 & -0.18 & -0.04 & 0.60 & 0.32 & 0.12 \\
\hline $\mathrm{NO}_{3}^{-}$ & 0.03 & -0.03 & 0.98 & -0.02 & 0.03 & -0.02 \\
\hline $\mathrm{SO}_{4}^{2-}$ & 0.30 & -0.03 & -0.24 & 0.73 & -0.02 & 0.06 \\
\hline $\mathrm{NH}_{4}^{+}$ & 0.02 & -0.07 & 0.98 & -0.03 & 0.08 & 0.00 \\
\hline $\mathrm{Na}^{+}$ & 0.22 & 0.36 & 0.21 & 0.71 & -0.21 & 0.16 \\
\hline $\mathrm{K}^{+}$ & -0.05 & 0.07 & 0.06 & 0.04 & 0.82 & -0.06 \\
\hline $\mathrm{Ca}^{2+}$ & 0.94 & 0.14 & -0.03 & 0.24 & 0.04 & -0.06 \\
\hline $\mathrm{Mg}^{2+}$ & 0.94 & 0.07 & -0.01 & 0.26 & -0.02 & -0.02 \\
\hline Eigenvalue & 6.21 & 2.53 & 2.05 & 1.59 & 1.20 & 1.10 \\
\hline$\%$ Total variance & 32.66 & 13.31 & 10.80 & 8.39 & 6.32 & 5.77 \\
\hline Cumulative \% & 32.66 & 45.97 & 56.77 & 65.16 & 71.48 & 77.25 \\
\hline
\end{tabular}

Significant factor loading in bold $>0.7$.

in their urine samples were measured and presented in Table 6. The concentration of $\mathrm{F}^{-}$in the buffalo and cattle urines was ranged from 18 - 52 and 26 - $58 \mathrm{mg} / \mathrm{L}$ with mean value of $31 \pm 4$ and $41 \pm 4 \mathrm{mg} / \mathrm{L}$, respectively. At least 7 - 10 folds higher $\mathrm{F}^{-}$content in the urine of animals was marked, may be due intake of higher dose of F-contaminated water and food. The higher fluorosis prevalence rate was observed in the cattle than buffalo, Figures 4-6. 
Table 6. Fluoride exposure in animals.

\begin{tabular}{|c|c|c|c|c|c|}
\hline S. No. & Animal & Color & Age, Yr & $\mathrm{F}^{-}, \mathrm{mg} / \mathrm{L}$ & Fluorosis \\
\hline 1 & Buffalo & B & 6 & 32 & $\mathrm{DF}$ \\
\hline 2 & Buffalo & B & 5 & 35 & DF \\
\hline 3 & Buffalo & B & 13 & 29 & $\mathrm{DF}$ \\
\hline 4 & Buffalo & $\mathrm{Br}$ & 8 & 40 & $\mathrm{DF}$ \\
\hline 5 & Buffalo & B & 14 & 37 & $\mathrm{DF}$ \\
\hline 6 & Buffalo & B & 5 & 35 & DF \\
\hline 7 & Buffalo & B & 5 & 18 & $\mathrm{DF}$ \\
\hline 8 & Buffalo & B & 7 & 29 & DF \\
\hline 9 & Buffalo & B & 5 & 22 & DF \\
\hline 10 & Buffalo & B & 8 & 45 & SK \\
\hline 11 & Buffalo & B & 5 & 29 & SK \\
\hline 12 & Buffalo & B & 5 & 21 & $\mathrm{DF}$ \\
\hline 13 & Buffalo & B & 6 & 19 & $\mathrm{DF}$ \\
\hline 14 & Buffalo & B & 5 & 22 & DF \\
\hline 15 & Buffalo & B & 9 & 52 & $\mathrm{TF}$ \\
\hline 16 & Buffalo & B & 7 & 19 & $\mathrm{TF}$ \\
\hline 17 & Buffalo & B & 7 & 33 & $\mathrm{TF}$ \\
\hline 18 & Buffalo & B & 6 & 35 & $\mathrm{TF}$ \\
\hline 19 & Buffalo & B & 8 & 27 & $\mathrm{TF}$ \\
\hline 20 & Buffalo & B & 15 & 31 & $\mathrm{TF}$ \\
\hline 21 & Cattle & W & 10 & 58 & SF \\
\hline 22 & Cattle & W & 8 & 40 & SF \\
\hline 23 & Cattle & $\mathrm{Br}$ & 8 & 39 & SF \\
\hline 24 & Cattle & $\mathrm{R}$ & 7 & 34 & SF \\
\hline 25 & Cattle & Gray & 5 & 32 & SF \\
\hline 26 & Cattle & WG & 6 & 36 & $\mathrm{HF}$ \\
\hline 27 & Cattle & WG & 11 & 56 & $\mathrm{HF}$ \\
\hline 28 & Cattle & G & 8 & 42 & $\mathrm{HF}$ \\
\hline 29 & Cattle & BW & 8 & 39 & $\mathrm{HF}$ \\
\hline 30 & Cattle & WG & 8 & 56 & $\mathrm{HF}$ \\
\hline 31 & Cattle & WG & 9 & 46 & HF \\
\hline 32 & Cattle & WG & 5 & 37 & $\mathrm{HF}$ \\
\hline 33 & Cattle & W & 8 & 58 & HF \\
\hline 34 & Cattle & W & 7 & 37 & HF \\
\hline 35 & Cattle & BW & 6 & 32 & $\mathrm{~L}$ \\
\hline 36 & Cattle & BW & 5 & 26 & $\mathrm{~L}$ \\
\hline 37 & Cattle & W & 5 & 34 & $\mathrm{~L}$ \\
\hline 38 & Cattle & W & 6 & 29 & $\mathrm{~L}$ \\
\hline 39 & Cattle & $\mathrm{Br}$ & 7 & 39 & $\mathrm{~L}$ \\
\hline 40 & Cattle & $\mathrm{Br}$ & 9 & 47 & $\mathrm{~L}$ \\
\hline
\end{tabular}

$\mathrm{DF}=$ Dental fluorosis, $\mathrm{SF}=$ Skin fluorosis, $\mathrm{TF}=$ Toe fluorosis, $\mathrm{HF}=$ Horn fluorosis, $\mathrm{L}=$ Lesion. 


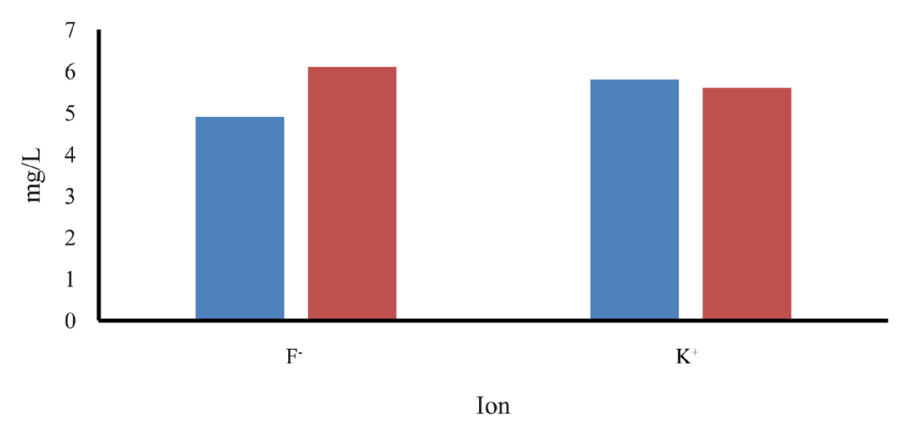

- Post Monsoon $\quad$ - Pre Monsoon

(a)

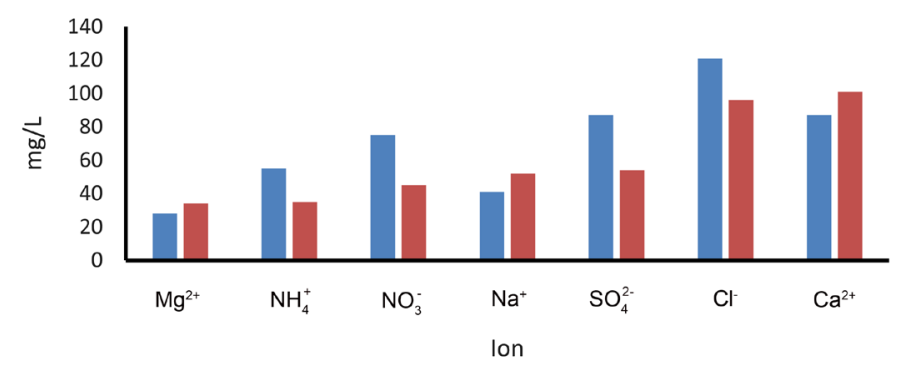

- Post Monsoon a Pre Monsoon

(b)

Figure 2. Seasonal variation of ionic concentration in post (a) and pre (b) monsoon period, 2014.

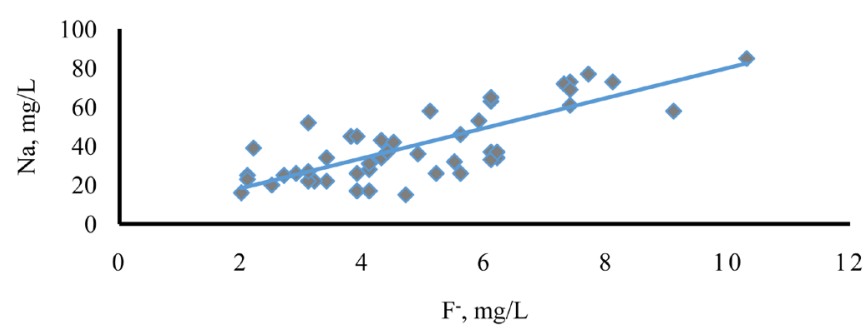

(a)

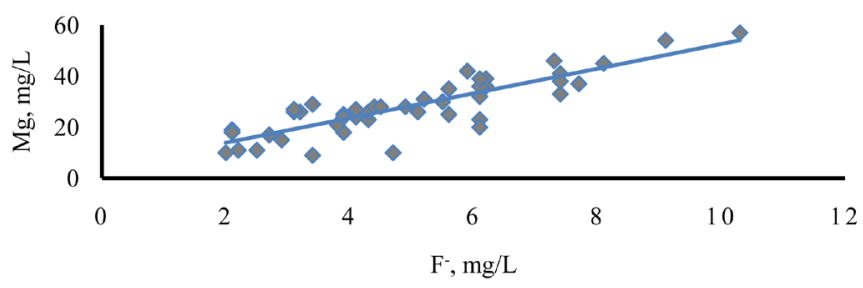

(b)

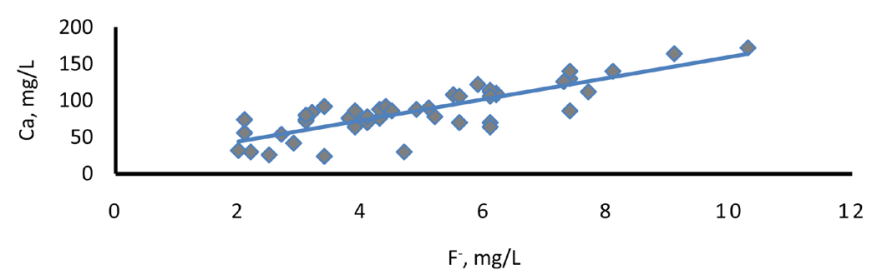

(c)

Figure 3. Correlation of $\mathrm{F}^{-}$with $\mathrm{Na}^{+}$(a), $\mathrm{Mg}^{2+}$ (b) and $\mathrm{Ca}^{2+}$ (c). 


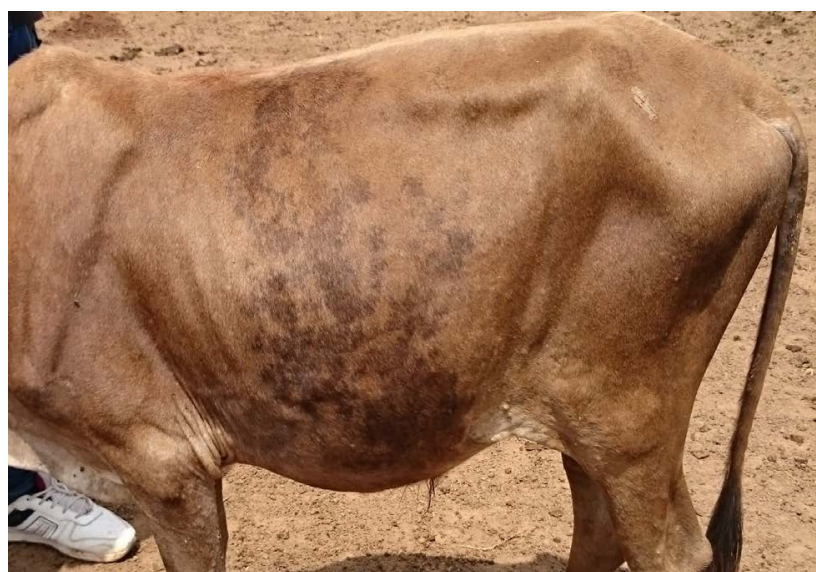

Figure 4. Skin lesson in cattle.

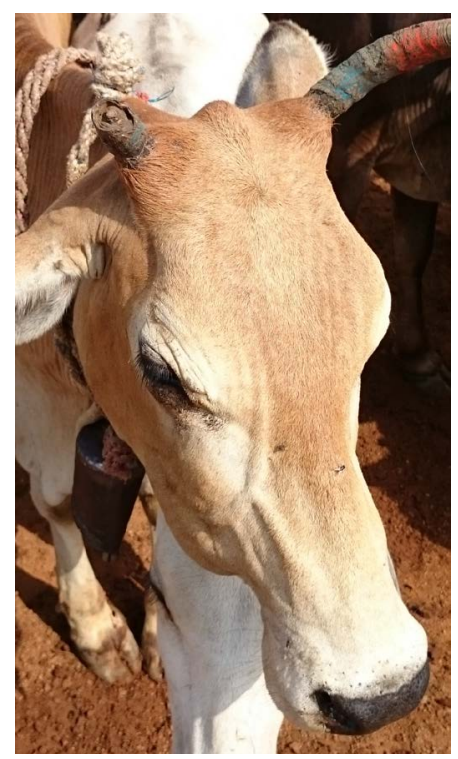

Figure 5. Horn fluorosis in cattle.

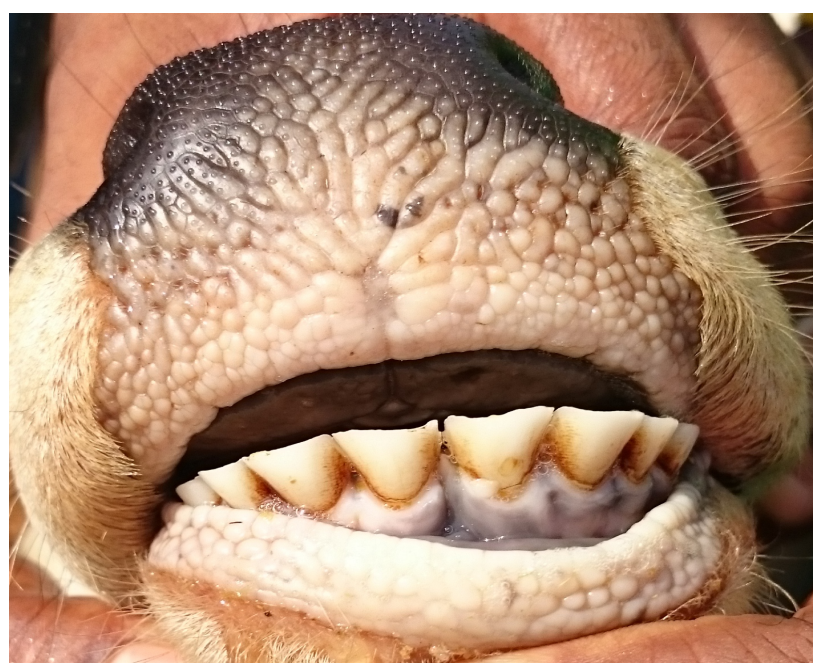

Figure 6. Dental fluorosis in buffalo. 


\section{Conclusion}

The groundwater of Dongargarh is contaminated with $\mathrm{F}^{-}$at dangerous levels due to mineralization of the bed rock $\mathrm{F}^{-}$in the water. The WQI index of water was found to be $\approx 100$, making water unsafe for dinking purposes. The $\mathrm{F}^{-}$levels in urine of cattle and buffalo were found several folds higher than recommended value of $4 \mathrm{mg} / \mathrm{L}$. Around 5\% domestic animals of the studied were suffered with different types of fluorosis.

\section{Acknowledgements}

We are thankful to the UGC, New Delhi for awarding Rajiv Gandhi Fellowship to one of the author: NSD.

\section{References}

[1] Saxena, V.K. and Ahmad, S. (2001) Dissolution of Fluoride in Groundwater: A Water-Rock Interaction Study. Environmental Geology, 40, 1084-1087. http://dx.doi.org/10.1007/s002540100290

[2] Datta, A.S., Chakrabortty, A., De Dalal, S.S. and Lahiri, S.C. (2014) Fluoride Contamination of Underground Water in West Bengal, India. Fluoride, 47, 241-248.

[3] Brindha, K., Rajesh, R., Murugan, R. and Elango, L. (2011) Fluoride Contamination in Groundwater in Parts of Nalgonda District, Andhra Pradesh, India. Environmental Monitoring and Assessment, 172, 481-492. http://dx.doi.org/10.1007/s10661-010-1348-0

[4] Arveti, N., Sarma, M.R., Aitkenhead-Peterson, J.A. and Sunil, K. (2011) Fluoride Incidence in Groundwater: A Case Study from Talupula, Andhra Pradesh, India. Environmental Monitoring and Assessment, 172, 427-443. http://dx.doi.org/10.1007/s10661-010-1345-3

[5] Reddy, A.G., Reddy, D.V., Rao, P.N. and Prasad, K.M. (2010) Hydrogeochemical Characterization of Fluoride Rich Groundwater of Wailpalli Watershed, Nalgonda District, Andhra Pradesh, India. Environmental Monitoring and Assessment, 171, 561-577. http://dx.doi.org/10.1007/s10661-009-1300-3

[6] Rao, N.S. (2009) Fluoride in Groundwater, Varaha River Basin, Visakhapatnam District, Andhra Pradesh, India. Environmental Monitoring and Assessment, 152, 47-60. http://dx.doi.org/10.1007/s10661-008-0295-5

[7] Suthar, S., Garg, V.K., Jangir, S., Kaur, S., Goswami, N. and Singh, S. (2008) Fluoride Contamination in Drinking Water in Rural Habitations of Northern Rajasthan, India. Environmental Monitoring and Assessment, 145, 1-6. http://dx.doi.org/10.1007/s10661-007-0011-x

[8] Vikas, C. (2009) Occurence and Distribution of Fluoride in Groundwaters of Central Rajasthan, India. Journal of Environmental Science and Engineering, 51, 169-174.

[9] Rao, N.S. (2011) High-Fluoride Groundwater. Environmental Monitoring and Assessment, 176, 637-645. http://dx.doi.org/10.1007/s10661-010-1609-y

[10] Hussain, I., Arif, M. and Hussain, J. (2012) Fluoride Contamination in Drinking Water in Rural Habitations of Central Rajasthan, India. Environmental Monitoring and Assessment, 184, 5151-5158. http://dx.doi.org/10.1007/s10661-011-2329-7

[11] Kundu, M.C. and Mandal, B. (2009) Assessment of Potential Hazards of Fluoride Contamination in Drinking Groundwater of an Intensively Cultivated District in West Bengal, India. Environmental Monitoring and Assessment, 152, 97-103. http://dx.doi.org/10.1007/s10661-008-0299-1

[12] Jha, S.K., Nayak, A.K. and Sharma, Y.K. (2010) Potential Fluoride Contamination in the Drinking Water of Marks Nagar, Unnao District, Uttar Pradesh, India. Environmental Geochemistry and Health, 32, 217-226. http://dx.doi.org/10.1007/s10653-009-9277-y

[13] Srikanth, R., Gautam, A., Jaiswal, S.C. and Singh, P. (2013) Urinary Fluoride as a Monitoring Tool for Assessing Successful Intervention in the Provision of Safe Drinking Water Supply in Five Fluoride-Affected Villages in Dhar District, Madhya Pradesh, India. Environmental Monitoring and Assessment, 185, 2343-2350. http://dx.doi.org/10.1007/s10661-012-2713-y

[14] Avishek, K., Pathak, G., Nathawat, M.S., Jha, U. and Kumari, N. (2010) Water Quality Assessment of Majhiaon Block of Garwa District in Jharkhand with Special Focus on Fluoride Analysis. Environmental Monitoring and Assessment, 167, 617-623. http://dx.doi.org/10.1007/s10661-009-1077-4

[15] Singh, C.K. and Mukherjee, S. (2015) Aqueous Geochemistry of Fluoride Enriched Groundwater in Arid Part of Western India. Environmental Science and Pollution Researches International, 22, 2668-2678. http://dx.doi.org/10.1007/s11356-014-3504-5

[16] Varadarajan, N. and Purandara, B.K. (2008) Fluoride Contamination in Ground Water of Malaprabha Sub Basin. Journal of Environmental Science and Engineering, 50, 121-126. 
[17] Sajil Kumar, P.J. (2012) Assessment of Fluoride Contamination in Groundwater as Precursor for Electrocoagulation. Bulletin of Environmental Contamination and Toxicology, 89, 172-175. http://dx.doi.org/10.1007/s00128-012-0638-3

[18] Vijay, R., Khobragade, P. and Mohapatra, P.K. (2011) Assessment of Groundwater Quality in Puri City, India: An Impact of Anthropogenic Activities. Environmental Monitoring and Assessment, 177, 409-418. http://dx.doi.org/10.1007/s10661-010-1643-9

[19] Banerjee, A. (2015) Groundwater Fluoride Contamination: A Reappraisal. Geoscience Frontiers, 6, 277-284. http://dx.doi.org/10.1016/j.gsf.2014.03.003

[20] Patel, K.S., Sharma, R., Sahu, B.L., Patel, R.K. and Matini, L. (2014) Ground Water Quality of Rajnandgaon City. Asian Journal of Water, Environment and Pollution, 11, 31-37.

[21] Dutta, R.K., Saikia, G., Das, B., Bezbaruah, C., Das, H.B. and Dube, S.N. (2006) Fluoride Contamination in Groundwater of Central Assam India. Asian Journal of Water, Environment and Pollution, 3, 93-100.

[22] Arif, M., Husain, I., Hussain, J. and Kumar, S. (2013) Assessment of Fluoride Level in Groundwater and Prevalence of Dental Fluorosis in Didwana Block of Nagaur District, Central Rajasthan, India. International Journal of Occupational and Environmental Medicine, 4, 178-184.

[23] Yadav, J.P., Lata, S., Kataria, S.K. and Kumar, S. (2009) Fluoride Distribution in Groundwater and Survey of Dental Fluorosis among School Children in the Villages of the Jhajjar District of Haryana, India. Environmental Geochemistry and Health, 31, 431-438. http://dx.doi.org/10.1007/s10653-008-9196-3

[24] Nayak, B., Roy, M.M., Das, B., Pal, A., Sengupta, M.K., De, S.P. and Chakraborti, D. (2009) Health Effects of Groundwater Fluoride Contamination. Clinical Toxicology (Philadelphia), 47, 292-295. http://dx.doi.org/10.1080/15563650802660349

[25] Suthar, S. (2011) Contaminated Drinking Water and Rural Health Perspectives in Rajasthan, India: An Overview of Recent Case Studies. Environmental Monitoring and Assessment, 173, 837-849. http://dx.doi.org/10.1007/s10661-010-1427-2

[26] Nayak, B., Mohan Roy, M., Das, B., Pal, A., Sengupta, M.K., De, S.P. and Chakraborti, D. (2009) Health Effects of Groundwater Fluoride Contamination. Clinical Toxicology, 47, 292-295. http://dx.doi.org/10.1080/15563650802660349

[27] Kotecha, P.V., Patel, S.V., Bhalani, K.D., Shah, D., Shah, V.S. and Mehta, K.G. (2012) Prevalence of Dental Fluorosis \& Dental Caries in Association with High Levels of Drinking Water Fluoride Content in a District of Gujarat. The Indian Journal of Medical Research, 135, 873-877.

[28] Marya, C.M., Ashokkumar, B.R., Dhingra, S., Dahiya, V. and Gupta, A. (2014) Exposure to High-Fluoride Drinking Water and Risk of Dental Caries and Dental Fluorosis in Haryana, India. Asia-Pacific Journal of Public Health, 26, 295-303. http://dx.doi.org/10.1177/1010539512460270

[29] Panday, A. (2010) Prevalence of Fluorisis in an Endemic Village in Central India. Tropical Doctor, 40, 217-219. http://dx.doi.org/10.1258/td.2010.100032

[30] Viswanathan, G., Gopalakrishnan, S. and Siva Ilango, S. (2010) Assessment of Water Contribution on Total Fluoride Intake of Various Age Groups of People in Fluoride Endemic and Non-Endemic Areas of Dindigul District, Tamil Nadu, South India. Water Research, 44, 6186-6200. http://dx.doi.org/10.1016/j.watres.2010.07.041

[31] Viswanathan, G., Jaswanth, A., Gopalakrishnan, S., Siva Ilango, S. and Aditya, G. (2009) Determining the Optimal Fluoride Concentration in Drinking Water for Fluoride Endemic Regions in South India. Science of the Total Environment, 407, 5298-5307. http://dx.doi.org/10.1016/j.scitotenv.2009.06.028

[32] Bhattacharya, S., Majumdar, T.J., Rajawat, A.S., Panigrahi, M.K. and Das, P.R. (2012) Utilization of Hyperion Data over Dongargarh, India, for Mapping Altered/Weathered and Clay Minerals along with Field Spectral Measurements. International Journal of Remote Sensing, 33, 5438-5450. http://dx.doi.org/10.1080/01431161.2012.661094

[33] Nielson, D.M. and Nielson, G. (2006) The Essential Handbook of Groundwater Sampling. CRC Press, Boca Raton. http://dx.doi.org/10.1201/9781420042795

[34] Nollet, L.M.L. and De Gelder, L.S.P. (2007) Handbook of Water Analysis. 2nd Edition, CRC Press, Boca Raton.

[35] BIS (2009) Drinking Water-Specification. 2nd Edition, Bureau of Indian Standards, New Delhi. http://bis.org.in/sf/fad/FAD25(2047)C.pdf

[36] WHO (2011) Guidelines for Drinking-Water Quality. 4th Edition, World Health Organization, Geneva. http://www.haceclick.com.uy/documentos/GuIa_OMS\%202011_4aEd.pdf

[37] Patra, R.C., Dwivedi, S.K., Bhardwaj, B. and Swarup, D. (2000) Industrial Fluorosis in Cattle and Buffalo around Udaipur, India. Science of the Total Environment, 253, 145-150. http://dx.doi.org/10.1016/S0048-9697(00)00426-5 